\title{
Solar cycle 24: An unusual polar field reversal
}

\author{
P. Janardhan ${ }^{1}$, K. Fujiki ${ }^{2}$, M. Ingale ${ }^{1}$, S. K. Bisoi ${ }^{3}$, and D. Rout ${ }^{4}$ \\ 1 Physical Research Laboratory, Astronomy \& Astrophysics Division, Navrangpura, Ahmedabad 380 009, India \\ e-mail: jerry@prl.res.in \\ 2 Institute for Space-Earth Environmental Research, Furocho, Chikusa Ward, Nagoya 464-8601, Japan \\ e-mail: fujiki@isee.nagoya-u.ac.jp \\ ${ }^{3}$ Key Laboratory of Solar Activity, National Astronomical Observatories, Chinese Academy of Sciences, Beijing 100012, PR China \\ e-mail: susanta@nao.cas.cn \\ 4 Physical Research Laboratory, Space \& Atmospheric Sciences Division, Navrangpura, Ahmedabad 380 009, India
}

Received 8 March 2018 / Accepted 3 August 2018

\begin{abstract}
Context. It is well known that the polarity of the Sun's magnetic field reverses or flips around the maximum of each 11 year solar cycle. This is commonly known as polar field reversal and plays a key role in deciding the polar field strength at the end of a cycle, which is crucial for the prediction of the upcoming cycle.

Aims. We aim to investigate solar polar fields during cycle 24, using measurements of solar magnetic fields in the latitude range $55^{\circ}-90^{\circ}$ and $78^{\circ}-90^{\circ}$, to report a prolonged and unusual hemispheric asymmetry in the polar field reversal pattern in solar cycle 24 . Methods. This study was carried out using medium resolution line-of-sight synoptic magnetograms from the magnetic database of the National Solar Observatory at Kitt Peak (NSO/KP), USA for the period between February 1975 and October 2017, covering solar cycles 21-24 and high-resolution line-of-sight synoptic magnetograms from the Michaelson Doppler Imager instrument onboard the Solar Heliospheric Observatory. Synoptic magnetograms using radial measurements from the Heliospheric Magnetic Imager instrument onboard the Solar Dynamics Observatory, covering solar cycle 23 and 24, were also used.

Results. We show that the southern solar hemisphere unambiguously reversed polarity in mid-2013 while the reversal in the field in the northern solar hemisphere started as early as June 2012, was followed by a sustained period of near-zero field strength lasting until the end of 2014, after which the field began to show a clear rise from its near-zero value. While this study compliments a similar study carried out using microwave brightness measurements which claimed that the field reversal process in cycle 24 was completed by the end of 2015 , our results show that the field reversal in cycle 24 was completed earlier that is, in late 2014. Signatures of this unusual field reversal pattern were also clearly identifiable in the solar wind, using our observations of interplanetary scintillation at $327 \mathrm{MHz}$ which supported our magnetic field observations and confirmed that the field reversal process was completed at the end of 2014.
\end{abstract}

Key words. Sun: activity - Sun: magnetic fields - Sun: photosphere - Sun: heliosphere - sunspots

\section{Introduction}

Reversal in the magnetic field polarity of planetary bodies that have a global magnetic field is a common phenomenon during which the north and south pole of the planet's magnetic field reverses and changes orientation. For example, the Earth shows a field reversal which occurs on time scales of millennia. Unlike the Earth however, the Sun's global dipole magnetic field flips or reverses polarity every 11 years around the maximum phase of each 11 year solar cycle. During the reversal, the polarity of the solar polar fields in both hemispheres reverses or changes to the opposite polarity. This process was first reported in the epoch making paper by Babcock (1961) after the first measurements of the Sun's polar magnetic fields in 1959 (Babcock 1959). Since then this phenomena has been extensively studied (Howard 1972; Makarov et al. 1983; Webb et al. 1984; Fox et al. 1998; Durrant \& Wilson 2003; Benevolenskaya 2004; Dikpati et al. 2004; Muñoz-Jaramillo et al. 2012) using synoptic magnetic charts, ground and space based magnetograms, observations of polar coronal holes and polar crown filaments.

The Sun's polar field at both poles attains its maximum at solar minimum, while it reduces and runs through zero during solar cycle maximum when the reversal of fields occurs at poles.
Polar fields have thus been a good indicator of the Sun's polar reversal. The time of solar polar field reversals in each hemisphere is identifiable as an unambiguous change in the sign of the polar fields or in other words, a clearly identifiable zero crossing of the polar fields in both hemispheres. In our earlier reported papers (Janardhan et al. 2010, 2011, 2015a,b; Bisoi et al. 2014a), we studied solar polar magnetic fields during solar cycles 21-23, using magnetic measurements from the National Solar Observatory, Kitt Peak (NSO/KP, USA) and the Michelson Doppler Interferometer on board the Solar and Heliospheric Observatory (SoHO/MDI; Domingo et al.1995). Polar magnetic fields from the solar photosphere extends out into the corona and beyond into the interplanetary medium through regions of the corona known as polar coronal holes. Large polar coronal holes begin to form during the late declining phase of a solar cycle, become very prominent at solar minimum and are the source of high-speed solar wind streams into the heliosphere (Krieger et al. 1973; Nolte et al. 1976; Zirker 1977). These large polar coronal holes are usually absent during the solar maximum phase when the polar field reversal process is ongoing and develop only months after the reversal (Kirk et al. 2009). They are, thus, another commonly used indicator of polar reversal process. 
Table 1. Estimates of the time of polar field reversals in solar cycle 24 as reported by different groups of researchers.

\begin{tabular}{|c|c|c|}
\hline Authors & $\begin{array}{c}\text { Reversal time } \\
\text { (north) }\end{array}$ & $\begin{array}{l}\text { Reversal time } \\
\quad \text { (south) }\end{array}$ \\
\hline Karna et al. (2014) & Jun. 2012 & - \\
\hline Sun et al. (2015) & Nov. 2012 & Mar. 2014 \\
\hline Pastor Yabar et al. (2015) & Feb. 2013 & Apr. 2014 \\
\hline Gopalswamy et al. (2016) & $\begin{array}{r}\text { Oct. } 2012 \\
\text { to Sent. } 2015\end{array}$ & Jun. 2014 \\
\hline This study & $\begin{array}{r}\text { Jun. } 2012 \\
\text { to Nov. } 2014\end{array}$ & Nov. 2013 \\
\hline
\end{tabular}

The reversal in the current solar cycle 24 has also been studied by many researchers (Svalgaard \& Kamide 2013; Karna et al. 2014; Bisoi et al. 2014a; Janardhan et al. 2015a; Sun et al. 2015; Gopalswamy et al. 2016). It is to be noted however, that the reported time of polar field reversal process for the current cycle 24 by the different researchers is different. Using polar coronal hole area measurements, deduced from data of the Helioseismic and Magnetic Imager (HMI) on board the Solar Dynamics Observatory (SDO; Lemen et al. 2012), the epoch of polar field reversal in the northern hemisphere, in cycle 24, was estimated to be mid-2012 (Karna et al. 2014). In another study, Sun et al. (2015) reported, using high cadence HMI 720s magnetograms, that the reversal of polar fields in the northern hemisphere occurred in November 2012, while the reversal in the southern hemisphere occurred in March 2014. Using line-of-sight HMI magnetograms for a 5 year period starting from April 2010, Pastor Yabar et al. (2015) reported the reversal times in the north and south to be Feb. 2013 and April 2014, respectively. More recently, using $17 \mathrm{GHz}$ microwave images and high latitudes prominence activity, Gopalswamy et al. (2016) reported, that the polar reversal in the southern hemisphere occurred around June 2014, while in the northern hemisphere the reversal was completed only by October 2015. The ambiguous nature of polar field reversal in cycle 24 is thus very clear as seen from Table 1, which summarises the various estimates of polar field reversal times for the northern and southern solar hemispheres by different workers. It must be noted here that taking into account the different methods used, the measurement uncertainties and the difficulty in assigning a given date to a process which is slowly changing with time, one can consider the reported times as homogenous if the difference is $\leq$ six months. From Table 1 it is clear that there is a difference of about a year between our estimate for the end of the reversal in the North and that of Gopalswamy et al. (2016).

In the present paper, we have extended our earlier studies (Janardhan et al. 2010, 2011, 2015a,b; Bisoi et al. 2014a) of solar polar fields using magnetic field measurements from NSO/KP, covering solar cycles 21-24, spanning the period from February 1975 to October 2017, to investigate the unusual manner in which the solar polar field reversal took place in cycle 24. Synoptic maps of solar wind velocities obtained using ground-based interplanetary scintillation measurements at $327 \mathrm{MHz}$ were also used in the present study to establish the development of polar coronal holes in order to investigate the polar reversal pattern in cycle 24 .

The onset of the current cycle 24 was delayed, due to the extended and deep solar minimum experienced in cycle 23 , with the first sunspots of the cycle 24 appearing only in March 2010 instead of the expected time of December 2008 (Jian et al. 2011). Also, solar cycle 24 is the fourth in a series of successively weaker cycles since cycle 21 , and is actually the weakest of the four. Solar cycle 23, not only experienced one of the deepest minima in the past 100 years but the peak smoothed sunspot number (V2.0) was 116 in April 2014, making it the weakest sunspot cycle since cycle 14, which had a smoothed sunspot number peak (V2.0) of $\sim 107$ in February 1906. It is known that the polar field strength at the end of a given cycle is determined by the amount of flux cancelled during polar field reversal, which is predominantly decided by the systematic tilt angle distribution of bipolar sunspot regions, in combination with processes of solar differential rotation and advection (Babcock 1961; Leighton 1969; Wang et al. 1989; Petrie 2012). It is, therefore, imperative that we try and understand how the complex nature of solar activity in cycle 24 has contributed to the solar polar field reversal process during cycle 24 .

\section{Data and methodology}

For computation of photospheric magnetic fields at different latitude ranges, we used medium-resolution line-of-sight synoptic magnetograms from both the NSO/KP database and the Synoptic Optical Long-term Investigation of the Sun facility (NSO/SOLIS). The synoptic magnetogram used were from February, 1975 to October, 2017, covering Carrington rotation (CR) CR1625-CR2195. Each synoptic map is generally made from several full-disk daily solar magnetograms observed over a Carrington rotation period covering 27.2753 days. These maps, available online as standard FITS files, contain photospheric magnetic fields in units of Gauss, in sine of latitude and longitude format of $180 \times 360$ pixels. Also, for a comparison of photospheric fields, obtained from medium-resolution NSO/KP synoptic magnetograms, we computed photospheric fields in the corresponding latitude ranges for cycle 24, using high-resolution radial synoptic magnetograms from the SDO/HMI. These maps have $1440 \times 3600$ pixels in sine of latitude and longitude format, covering CR2096-CR2194 (from April 2010 to Aug. 2017). For cycle 23, we used high-resolution line-of-sight synoptic magnetograms from the SoHO/MDI, available as maps having $1440 \times$ 3600 pixels in sine of latitude and longitude format, covering CR 1911-CR2080 (from April 1996 to February 2009). In order to compare the results from NSO/KP, we degraded (by averaging) the resolution of both the SoHO/MDI and SDO/HMI to the resolution of NSO/KP. In addition, we also used line-of-sight measurements of ten-day averaged values of polar fields obtained at the Wilcox Solar Observatory (WSO) in the latitude range poleward of $55^{\circ}$, covering solar cycles $21-24$. It is to be noted however, that due to the inclination of the Earth's orbit to the Sun's equator, one of the poles will not be clearly visible. The data at $\mathrm{NSO} / \mathrm{KP}$ and SOHO/MDI is made available online, to the general user, after having been corrected for this effect, while the $\mathrm{SDO} / \mathrm{HMI}$ data is available without this correction being incorporated. We therefore computed polar fields for SDO/HMI in the latitude range $55^{\circ}-78^{\circ}$ leaving out the pole-most regions. Additionally, in order to eliminate yearly geometric projection effects at the poles due to the rotation of Earth on the measured line-of-sight fields, we used a 1.58 year low pass filter to filter WSO polar fields. Thus, the obtained filtered polar fields are free from the effect of any kind of annual periodicities, which if not removed may result in showing multiple zero-crossing of the polar fields during solar polar field reversal thereby causing confusion about the occurrence of multiple polar field reversals.

For each synoptic map, photospheric magnetic fields were first estimated for each of the 180 arrays in sine of latitude by taking a longitudinal average of the entire 360 array of 
Carrington longitude. Thus, each CR map, previously in the form of a $180 \times 360$ array, was reduced to an array of $180 \times 1$. Thereafter, for computing magnetic fields at selected latitude ranges, we averaged the longitudinal strip of $180 \times 1$ over appropriate latitude ranges. For the present study, we selected three latitude zones: toroidal fields, polar fields, and polar cap fields in the latitude range $0^{\circ}-45^{\circ}, 55^{\circ}-90^{\circ}$, and $78^{\circ}-90^{\circ}$, respectively. It is, however, to be noted that the latitudinal range defined as polar has been arbitrary with different researchers using different latitude ranges for estimation of polar fields such as poleward of $45^{\circ}$ (Janardhan et al. 2011; Bisoi et al. 2014a), 55 (Wilcox Solar Observatory polar fields ${ }^{1}$ ), $60^{\circ}$ (de Toma 2011; Gopalswamy et al. 2012, 2016; Sun et al. 2015), and $70^{\circ}$ (Muñoz-Jaramillo et al. 2012). For the present study, in order to compute polar fields, we preferred the latitude range between $55^{\circ}$ and $90^{\circ}$, which is same as the latitude range used to measure polar fields at WSO. Similar latitude ranges $\left(>50^{\circ}\right)$ have been used by Karna et al. (2014) in order to compute polar coronal hole area to report the polar reversal process in cycle 24 . Altrock (2011) reported the appearance of the Fe XIV emission feature in the corona at latitudes $>50^{\circ}$, prior to the solar cycle maximum, which started to drift to the poles over time and which he called as rush-to-the-poles. The author suggested that the solar maximum usually occurs when a linear fit to the rush-to-the-poles data reaches $76^{\circ} \pm 2^{\circ}$. We, therefore, also computed polar cap fields in the latitude range $78^{\circ}-90^{\circ}$ to study the polar field reversal process at the polemost latitudes. Earlier, Benevolenskaya (2004) used SDO/HMI data in the latitude range poleward of $78^{\circ}$ to report the behaviour of polar fields during polar field reversal in cycle 23. In our earlier work (Janardhan et al. 2010), we used also the same latitude range to report the correlation of meridional flow speed with polar fields during cycle 23.

For studying solar wind signatures due to outflows from polar coronal hole regions, we constructed synoptic velocity maps using $327 \mathrm{MHz}$ interplanetary scintillation (IPS) observations from the three station IPS facility of the Institute for Space-Earth Environmental Research (ISEE), Nagoya University, Nagoya, Japan. IPS is the method of studying random temporal variations of the signal intensity of distance extra-galactic radio sources using ground based telescopes, typically observed at meter wavelengths (Hewish et al. 1964; Janardhan \& Alurkar 1993; Ananthakrishnan et al. 1995; Janardhan et al. 1996; Moran et al. 2000; Balasubramanian et al. 2003; Bisoi et al. 2014b). A tomographic technique (Kojima et al. 1998) was used to produce synoptic solar wind velocity maps. Polar coronal holes, as the name suggests, are identifiable in the polar regions of the synoptic maps as large, extended regions of high velocity solar wind outflows, appearing after polar field reversal.

\section{Results and discussions}

\subsection{Photospheric signatures}

The upper panels in Fig. 1 show, by red filled dots with $1 \sigma$ error bars, the signed value of the variation of polar fields computed in the latitude range $55^{\circ}-90^{\circ}$, in the northern (top) and southern (bottom) hemispheres, during solar cycles 21-24. Each cycle is demarcated by a vertical dashed line. A smoothed curve through the data points in both hemispheres is shown by a solid black line with $1 \sigma$ error bars (pink), after using a 1.58 year filter so as to remove projection effects caused by the rotation of the Earth. The

http://wso.stanford.edu/Polar.html time of solar polar field reversals in each hemisphere, identifiable as a change in the sign of the field in other words, a clearly identifiable zero crossing of the field, is shown by small vertically orientated solid blue lines from cycles 21-24 and labelled with the month and year of the reversal. The lower two panels show a blown up portion of Fig. 1 only for solar cycle 24 . For comparison, shown in blue are the filtered polar fields from WSO using the 1.58 year filter and the signed values of polar field strengths from $\mathrm{SDO} / \mathrm{HMI}$, in green, in the lower two panels of Fig. 1. As mentioned earlier, it is to be noted that polar fields at WSO are usually measured in the latitude range $55^{\circ}-90^{\circ}$. It is evident from the variation of fields as shown by the smoothed curves of NSO/KP and WSO that their behaviour is similar. Thus, medium-resolution $\mathrm{NSO} / \mathrm{KP}$ magnetograms are still useful in studying the nature of the large scale polar fields. It can also be seen from Fig. 1 that the time of solar polar field reversals in each solar hemisphere, during cycle 21-24, for NSO/KP and WSO are both around the same time. Also, overplotted by a solid curve in green in the lower panels of Fig. 1 are the polar fields from SDO/HMI, in the latitude range $55^{\circ}-78^{\circ}$, for cycle 24 . It can be seen that the nature of polar fields and the time of reversal in cycle 24 , as shown by smoothed curves in black, blue, and green in the lower two panels, are by and large similar for the NSO/KP, WSO and the SDO/HMI data. The error bars have been omitted in the lower two panels to reduce clutter and enhance clarity.

The hemispheric asymmetry in polar field reversal is clearly evident from Fig. 1 for Cycles 21-24. However, unlike the previous cycle 23 , the polar field reversal in cycle 24 shows an unusual nature. It can be seen from Fig. 1 that the southern hemisphere underwent a clean, clearly identifiable field reversal in Nov. 2013. The reversal in the northern hemisphere, on the other hand, started in June 2012 and continued showing an extended near-zero field condition until November 2014, indicating that the completion of reversal of polar fields in the north in cycle 24 had not yet taken place. The fields in the northern hemisphere showed a rise after November 2014, indicating the completion of polar field reversal at that time. The WSO polar fields also show two clearly identifiable changes in the sign of the field for the northern hemisphere in cycle 24 with the first or initial reversal starting as early as in June 2012 and the second change in the sign of the field occurring almost 2 years later in November 2014 , indicating the completion of the field reversal at that time. Such a field reversal pattern, separated by two years between the two hemispheres and the prolonged zero-field conditions delaying the completion of reversal in one of the hemispheres by more than two years is very unusual, as field reversals usually occur in both the hemispheres within six months to a year of each other, and thereby completing the reversal process. This pattern, as observed in cycle 24, is clearly unusual as is apparent in Fig. 1 from field reversals seen in cycles 21, 22 and 23.

Other researchers have also reported similar hemispheric differences in polar reversal in cycle 24 . As mentioned earlier, it is to be noted that Karna et al. (2014) reported the start of the northern hemisphere reversal in mid-2012 using polar coronal hole areas and EUV emission in the corona above latitudes of $50^{\circ}$. Using polar fields computed from HMI data poleward of $60^{\circ}$, Sun et al. (2015) reported a reversal in the north in late 2012 and a reversal in the south in early 2014. The use of different latitude ranges can result in the difference of the time of reversal of polar fields, which is evident from Fig. 2. The upper panels in Fig. 2 show the variation of polar fields computed in the latitude range poleward of $45^{\circ}, 55^{\circ}$ and $60^{\circ}$, for the northern and southern hemispheres. It is clear from the upper panels of Fig. 2 that the overall behaviour of polar fields is the same whether one con- 

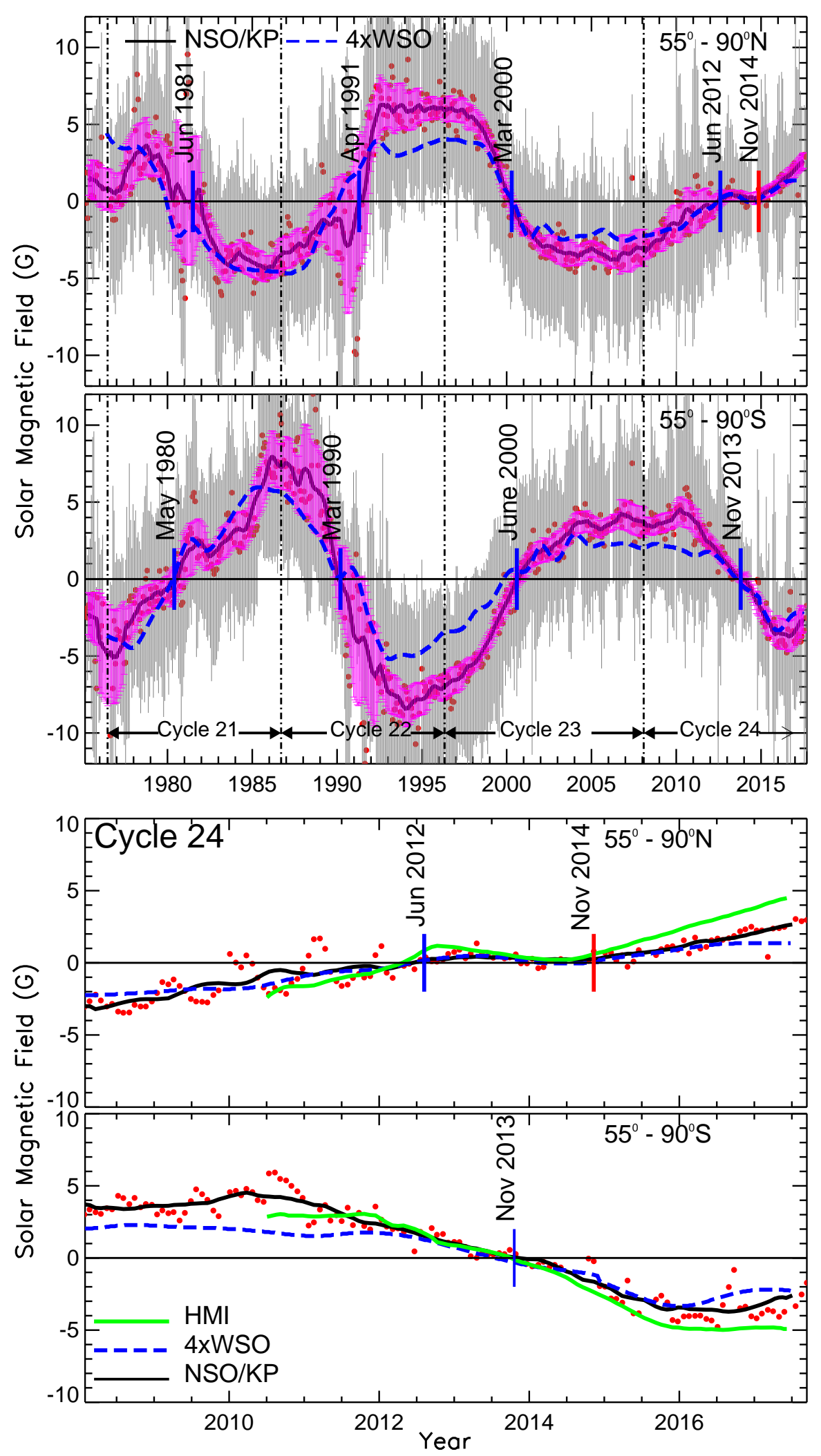

Fig. 1. Upper two panels show, by the filled dots (red) with $1 \sigma$ error bars (gray), signed values of polar field strengths from NSO/KP, in the latitude range $55^{\circ}-90^{\circ}$, in the solar northern (top) and southern (bottom) hemispheres spanning period covering solar cycles 21-24, each of which is demarcated by vertical dashed lines. A smoothed solid black curve with $1 \sigma$ error bars (pink) is drawn through the data points. Also, overplotted by a dashed blue curve is the signed values of polar field strengths from WSO. In order to eliminate yearly geometric projection effects at the poles due to the rotation of the Earth on the measured line-of-sight fields, a 1.58 year (or $20 \mathrm{nHz}$ ) low pass filter was used to filter WSO polar fields. The time of reversal of polarity in each hemisphere, determined from $\mathrm{NSO} / \mathrm{KP}$ smoothed curve, is marked by small blue solid lines in each panel, while the time of completion of polar reversal in the solar north is indicated by a small red solid line in cycle 24. Lower two panels show only cycle 24 , and to avoid clutter and enhance clarity, error bars have been left out. Overplotted by a solid green curve, in the lower two panels, is the signed values of polar field strengths from SDO/HMI. In cycle 24, the southern hemisphere underwent a clean unambiguous reversal in November 2013, while the northern hemisphere has shown an extended zero-field condition after the first reversal in June 2012. The reversal in the northern hemisphere was completed only by November 2014 . siders the latitude range poleward of $45^{\circ}, 55^{\circ}$ or $60^{\circ}$. The only difference that one sees is the different times of zero-crossing of the field during polar field reversal in each cycle. It is however to be noted that zero-crossing of the field usually occurs earlier for the fields at the lower latitude than the higher latitude. This can be understood from the fact that the cancellation of the opposite fluxes usually occurs first at lower latitudes. So the zonally averaged polar fields estimated over different latitude ranges will have different times for polar reversal. Surprisingly though, as evident from the lower panels of Fig. 2, the recovery of polar fields for all the latitude ranges, from the prolonged zero-field condition in the northern hemisphere in cycle 24 , started nearly around the same period, in November 2014, as indicated by a small blue vertical line in the lower panel of Fig. 2. 

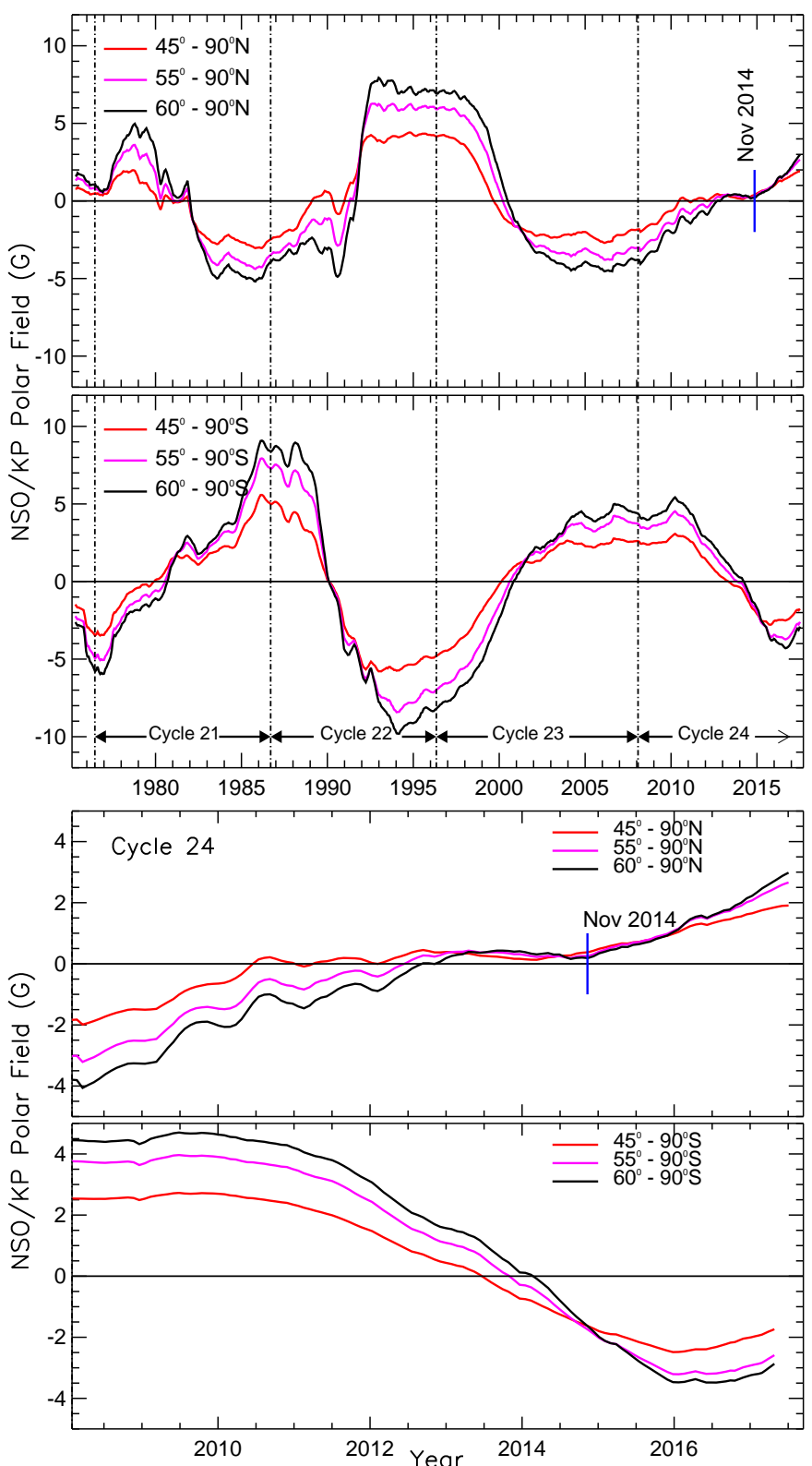

Fig. 2. Upper panels: comparison of the signed values of polar field strengths, obtained from line-of-sight NSO/KP magnetograms, in the latitude ranges, $45^{\circ}-90^{\circ}, 55^{\circ}-90^{\circ}$, and $60^{\circ}-90^{\circ}$ for the northern (top) and southern hemisphere (bottom), for the period February 1975November 2016, covering solar cycles 21-24. The reversal of polar fields is clearly seen from the zero-crossing of fields around the solar maximum phase of each cycle. Lower panels: blow up portion for cycle 24 . The extended zero-field conditions of polar reversal in the northern hemisphere is apparent at all latitude ranges, which is completed only around November 2014 as indicated by a small blue solid line in cycle 24.

In an another study of polar field conditions in cycle 24, using $17 \mathrm{GHz}$ microwave images obtained by the Nobeyama Radio Heliograph (NoRH), Gopalswamy et al. (2016) stated that the extended zero field condition in the solar northern hemisphere lasted until October 2015. The authors showed that there was an absence of microwave brightness enhancement at the northern polar region for three years following 2012, indicating a zero field condition in the northern hemisphere until 2015. It was also shown (Gopalswamy et al. 2016) that during this zero field period for three years, that is, until 2015 the microwave brightness enhancement showed undulating signatures which were interpreted as polar surges of alternating polarities reaching the north polar region. The polar surges are magnetic flux channels which can be best viewed in a magnetic butterfly diagram. We therefore constructed a magnetic butterfly diagram, for the period from February 1975 to December 2016, using NSO/KP magnetic field observations, in order to investigate the polar surges.

Figure 3 (top) shows such a zonally averaged magnetic field butterfly diagram constructed after removing the strong magnetic field regions, typically associated with sunspot regions in the equatorial belt in both hemispheres, up to about $35^{\circ}$ in latitude. As stated earlier, the original synoptic magnetogram data is a two dimensional map with 360 pixels in longitude and 180 pixels in Sine latitude. Each map was first re-gridded from sine latitude to latitude with a spatial resolution of one degree. A longitudinal average of the magnetic field in each latitude was then taken by considering only the data points with weak magnetic field strength $(|B| \leq 10 \mathrm{G})$. The strong magnetic fields associated with active regions and sunspots were therefore suppressed. The resulting magnetic butterfly diagram reproduces the polar surges with much more clarity and with higher contrast. Figure 3 (bottom) displays temporal profiles of the mean magnetic field in every $10^{\circ}$ bin in latitude obtained from the butterfly diagram in the upper panel. The red and blue points on the profiles (in gray), denote positive and negative polarity, respectively. A reversal of the polar field will be seen as a change in colour from red to blue and vice-versa, which is apparent in solar cycles 21-23. However, the polar reversal in cycle 24 shows an unusual pattern. The polarity reversal in cycle 24 can be noticed from a change of the polarity of from blue (red) points to red (blue) points, respectively, in the northern (southern) polar region above the latitude $>55^{\circ}$.

For an enhanced view, Fig. 4 shows the zonally averaged magnetic butterfly diagram (upper panel) and temporal profiles of mean magnetic fields (lower panel) only for cycle 24. As against the normal pattern of multiple surges of one polarity, it is evident from the butterfly diagram in Fig. 4 that there are multiple instances of surge activity of both polarities (positive and negative) in the northern hemisphere in cycle 24 while the polar surges in the southern hemisphere in cycle 24 are of same polarity (negative only). This is also clearly established from the profiles in Fig. 4 (bottom) which depict multiple changes of polarity (red to blue and blue to red) in the northern hemisphere at latitudes poleward of $>55^{\circ}$, while the southern hemisphere showed a clean reversal of polarity from positive (red) to negative (blue). The first poleward surge (of positive polarity) in the north, N1, was seen sometime around 2010, when the reversal in the north had begun changing polarity to positive. About a year later, the second poleward surge, N2 (of negative polarity) appeared, reversing the polarity to negative again. This pattern has repeated with subsequent surges N3, N4, N5 and N6 of alternate polarity until the largest and continuing polar surge N7 of positive polarity appearing in mid-2012, when finally the reversal in the north had occurred. However, the re-occurrence of alternate polarity kept the zero-field condition in the north until late 2014, when a large surge of magnetic flux, N8 finally completed the reversal in the north. The change of polarity in the southern hemisphere is comparatively very clean and was completed in late 2013. Multiple large surge activity in the south, indicated by S1, S2, S3, S4, and $\mathrm{S} 5$ of same polarity except for $\mathrm{S} 1$, are clearly noticeable in cycle 24 which establishes the clean reversal in the southern hemisphere. It is, thus, clear from Fig. 4 that the multiple surges of alternate polarity contributed to the prolonged zero-field conditions and the delayed field reversal in the northern hemisphere. As stated earlier, Gopalswamy et al. (2016) reported that the extended zero field condition in the northern hemisphere in cycle 24 lasted until 

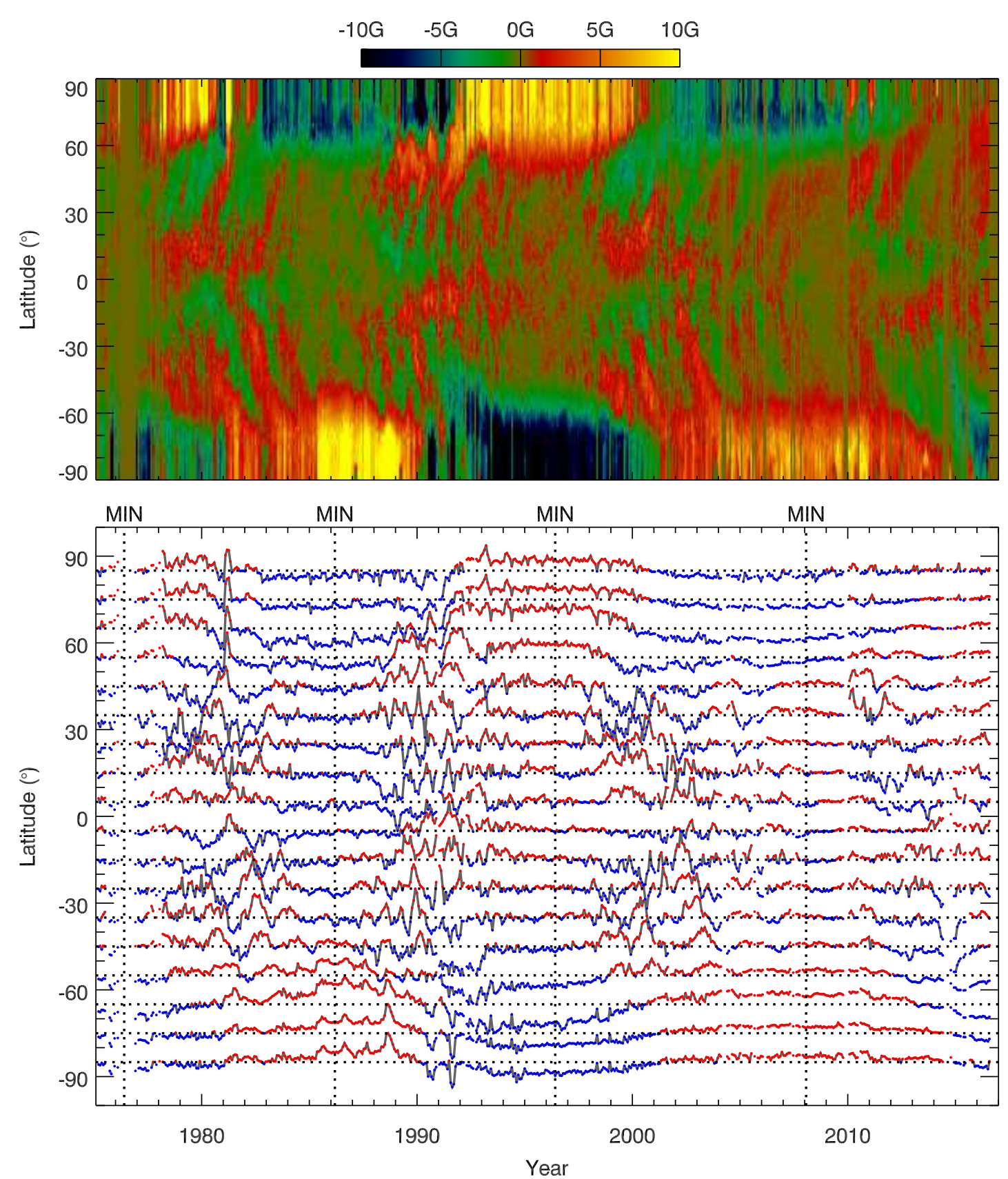

Fig. 3. Upper panel: zonally averaged magnetic diagram produced after re-gridding individual synoptic magnetograms from sine latitude to latitude with a spatial resolution of one degree and then taking longitudinal average of the magnetic field in each latitude by using only the data points with weak magnetic field strength $(|B|<10 \mathrm{G})$ to suppress the effects of sunspots in the mid-latitudes and to reproduce the magnetic flux transport to the poles with higher contrast. Lower panel: temporal profiles of the mean magnetic field in every $10^{\circ}$ latitude bin obtained from the butterfly diagram in the upper panel, with the red and blue points denoting positive and negative polarity, respectively.

October 2015, by which time the field reversal process in cycle 24 was complete. Our magnetic field observations, on the other hand, show that the prolonged zero field condition in the northern hemisphere in cycle 24 existed only until late 2014 when the field reversal process was finally completed in the north. The delay in completion of polar reversal can be understood from the arrival of polar surges of alternate polarity in the polar cap regions until late 2014.

\subsection{Polar cap fields and meridional flow speeds}

From Fig. 4, it is apparent that, in cycle 24, polar surges or tongues of magnetic flux channels were seen to be extended up to the polar cap latitudes of $\sim 75^{\circ}$. It is thus expected, that the poleward transport of magnetic flux in cycle 24 can be extended all the way to the poles. In fact, it is not uncommon as we have seen this during cycle 22 and 23 when such flux transport reached up to the latitudes of $\sim 60^{\circ}$ (Dikpati et al. 2010). In our earlier work (Janardhan et al. 2010), we used polar cap fields in the latitude range between $78^{\circ}$ and $90^{\circ}$ and showed a very good correlation between polar cap fields with the magnetic flux transport for cycle 23 . It is, therefore, necessary to verify the polar field variations in the polar cap regions beyond $\sim 75^{\circ}$. Figure 5 shows the unsigned values of polar cap field strengths, in the latitude range $78^{\circ}-90^{\circ}$, obtained from NSO/KP, for the northern (first panel) and southern (fourth panel) hemispheres, covering solar cycles 23 

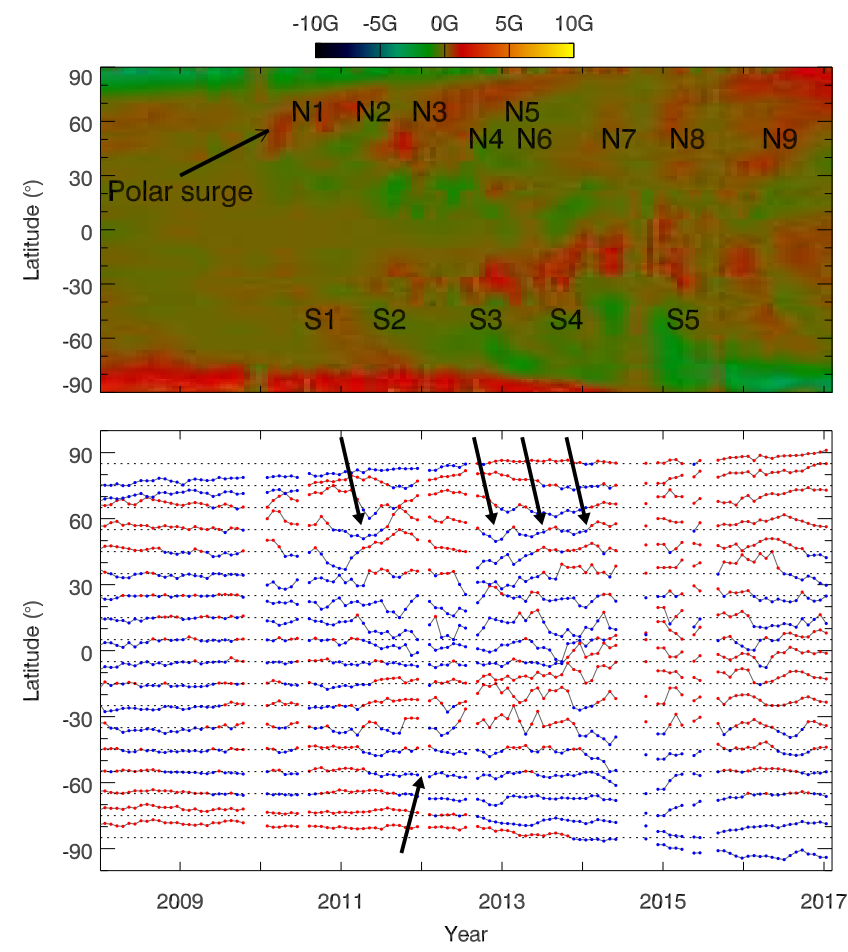

Fig. 4. Zonally averaged magnetic diagram (upper panel) and temporal profiles of mean magnetic fields (lower panel) same as shown in Fig. 3, but only for cycle 24 . The zonally averaged magnetic diagram is shown with magnetic field values saturated at $10 \mathrm{G}$. The polar surges in the north are numbered from N1 to N9, and that in the south are numbered from S1 to S5. The polarity changes in mean magnetic field profiles in the north and the south are indicated by arrows. Multiple arrows in the north indicate multiple change in polarity in the north compared to a single clean change in polarity in the south.

and 24. For comparison, the polar cap field strengths obtained from high-resolution data from SoHO/MDI (dashed blue curve) are overplotted at latitudes poleward of $78^{\circ}$ for cycle 23 and from HMI (dotted green curve) in the latitude range $60^{\circ}-78^{\circ}$ for cycle 24, respectively. It is evident from Fig. 5 that the polar field strength in the north reached its minimum values much earlier than the south showing the hemispheric phase shifts in polar field reversal process in cycle 24 . The polar fields after running through the minimum value recovers slowly in the northern hemisphere and the recovery of minimum condition has been completed only in late 2014 , as indicated by a small red vertical line. Though the polar cap fields in the southern hemisphere showed a faster recovery from minimum condition indicating the completion of reversal in the south occurring sometime in mid-2014. The time of completion of field reversal in both the hemispheres is around the similar period as the change of polarity at the polar cap latitudes from magnetic butterfly diagram shown in Fig. 4.

The second panel (northern hemisphere) and third panel (southern hemisphere) of Fig. 5, shown by a solid red curve, shows the variation of toroidal fields or photospheric fields in the latitude range $0^{\circ}-45^{\circ}$, derived from NSO/KP data, confined to the sunspot activity belt area. The hemispheric smoothed sunspot number (SSN) is overplotted in both the panels shown by a solid black curve. The hemispheric phase shifts in solar activity peak is evident from the toroidal fields for both cycle 23 and 24 . In cycle 23 , a double peak solar maximum is apparent for both the hemispheres, which is not the case in cycle 24 . The double peak maximum is seen only in the northern hemisphere, while the southern hemisphere peaks only once in June 2014. It may noted

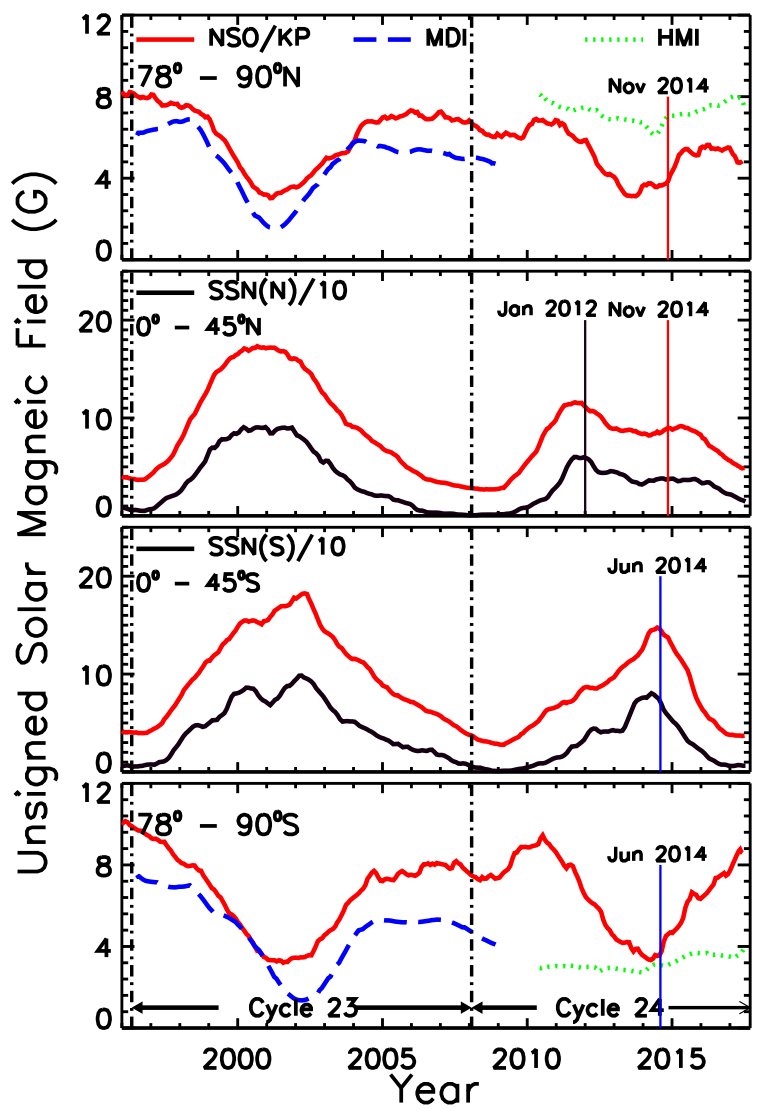

Fig. 5. Panels (from top to bottom): unsigned values of solar magnetic fields, obtained from NSO/KP (solid curve in red), SoHO/MDI (dashed curve in blue), and HMI (dotted curve in green) magnetograms, in the latitude ranges, poleward of $78^{\circ}$ in the north, $0^{\circ}-45^{\circ}$ in the north, $0^{\circ}-45^{\circ}$ in the south, and poleward of $78^{\circ}$ in the south, respectively, for solar cycles 23-24. The hemispheric smoothed sunspot number is shown in solid black curve overplotted in the second (north) and third (south) panels. The hemispheric asymmetry in solar activity in cycle 24 is evident with the south showing one activity peak (Jun 2014), while the north showing double activity peaks (Jan. 2012 and Nov. 2014). It is also clear from the first and fourth panels that the polar reversal process in the north in cycle 24 is slow and seems to be completed in Nov. 2014, while in the south, it is faster and has been completed in early 2014 . The occurrence and completion of polar reversal process happening around the solar cycle maximum is evident from the figure.

that the reversal in the south has been completed during the same period as seen in the fourth panel of Fig. 5. The first peak in the north occurred $\sim 2$ years earlier than in the south. However, the second peak occurred in Nov. 2014 when the reversal in the north was finally completed as observed in the first panel of Fig. 5. It is, thus, clear that the prolonged zero-field conditions in polar cap fields is linked to solar magnetic activity in the sunspot belt zone. Furthermore, it is evident that the polar field strength in cycle 24 during solar decline phase is much weaker than that in cycle 23. The good correlation between the polar cap field strength and the magnetic flux transport implies that the meridional flow speed could be much faster during the minimum of cycle 24 than the minimum in cycle 23 . This, according to surface flux transport models, can produce weaker polar fields during the minimum of cycle 24 . The prolonged zero-field condition in the northern hemisphere could be due to a faster meridional flow speed leading to more flux cancellation at the poles due to transport of more opposite magnetic flux from the sunspot belt region. 


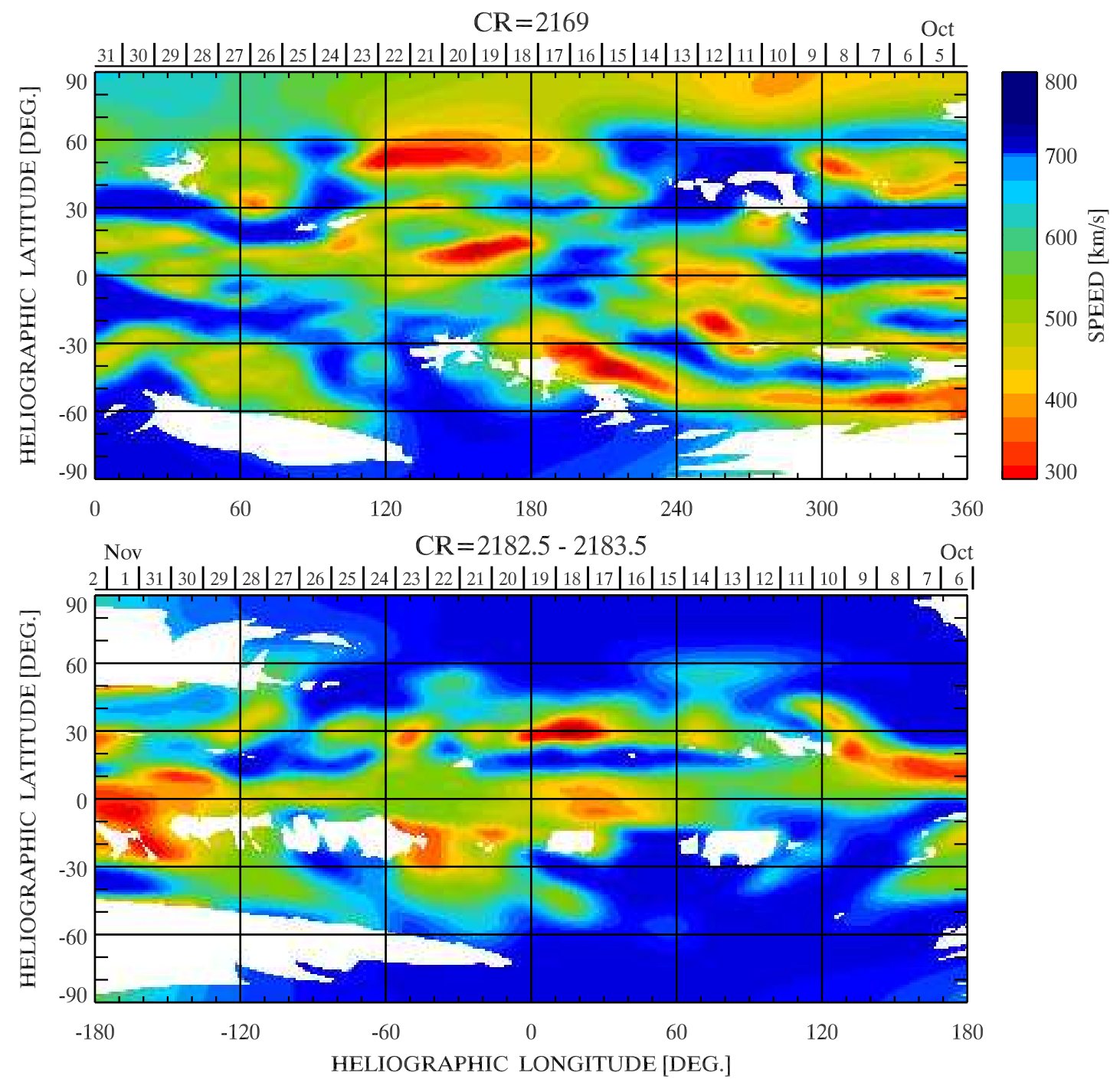

Fig. 6. Synoptic velocity maps for Carrington rotation 2169 in October 2015 (upper panel) and October 2016 (lower panel). The large polar coronal hole in the southern hemisphere is apparent but its counterpart in the northern hemisphere has not formed in as of October 2015 while it is fully developed in October 2016, a year later.

\subsection{Heliospheric signatures}

The heliospheric signatures of this extremely unusual polar field reversal pattern in cycle 24 can be seen in Fig. 6 which shows a synoptic map of solar wind velocities for Carrington rotation 2169 covering the period 04-31 October 2015 (upper panel) and for Carrington Rotations 2182.5-2183.5 covering the period 06 October 2016-02 November 2016 (lower panel). It is well known that polar coronal holes, which are the source regions of high speed solar wind (Krieger et al. 1973; Nolte et al. 1976; Zirker 1977), begin to develop in both solar hemispheres after the field reversal takes place. Nearly four decades of solar wind observations from the three-station IPS facility at ISEE, Japan, have shown that polar coronal holes recover and become fully developed $\sim 2$ years after the field reversal (Fujiki et al. 2016) and continue to expand and grow in area, as the minimum approaches. Fujiki et al. (2016) have in fact used an automated procedure to construct a database of 3335 coronal holes appearing between 1975 and 2015 and their study shows that the high latitude coronal holes recover in about 1.5 to 2 years after solar maximum. The synoptic map in the lower panel of Fig. 6 (06 Oct.-02 Nov. 2016) shows large, extended regions of high velocity solar wind outflows (in blue), or polar coronal holes, in the region poleward of $60^{\circ}$ in both hemispheres. It is clear from Fig. 6 (upper panel: 04-31 Oct. 2015) that a well defined polar coronal hole was present in the southern hemisphere by October 2015, 2 years after the field reversal in the south, that took place in Nov. 2013, while it had not developed in the northern hemisphere until a year later in Oct. 2016, as evident from Fig. 6 (lower panel). This is consistent with the asymmetric field reversal pattern seen in the northern and southern hemisphere in cycle 24, as shown in Fig. 1, which shows the completion of polarity reversal of fields in the southern hemisphere in late 2013 and in the northern hemisphere in late 2014. The completion of polar reversal would have been followed by the development of the polar coronal hole in both the hemisphere. Since the completion of reversal occurred at different times in the two hemispheres, we see a difference in time of appearance of fully developed polar coronal holes in the two hemispheres.

\section{Summary and conclusions}

We have examined the polar field reversal process from solar cycles 21-24, spanning the period between Jan. 1975 and Dec. 
2016, using magnetic field measurements from NSO/KP and the constructed magnetic butterfly diagrams which clearly depict the field reversal during each solar cycle. Our study highlights the unusual nature and the significant hemispheric asymmetry of the field reversal pattern in solar cycle 24 by examining high latitude solar magnetic fields, poleward of $55^{\circ}$ and $78^{\circ}$. The current study shows that the field reversal in the northern solar hemisphere was completed only by November 2014 while the southern hemisphere underwent a reversal in November 2013. However, a recent study (Gopalswamy et al. 2016), suggested that this process was completed only by the end of 2015, a full year later than our estimate from the present study. It is to be noted that these authors used $17 \mathrm{GHz}$ microwave images to show the absence of microwave brightness enhancement in the polar region during the prolonged zero-field polar field conditions in the northern hemisphere following 2012 until late 2015. The difference in completion of polar field reversal in the northern hemisphere is understood from the fact that the $17 \mathrm{GHz}$ microwave emission is generally observed in the lower corona or at best in the chromospheric region, but not at the photospheric level. However, our study of the polar reversal is at the photosphere and is therefore better in pinpointing the time of completion of the polar reversal in the solar northern hemisphere. Our study shows that the reversal occurred much earlier, in late 2014. Confirmation of our results also came from an entirely different study aimed at examining the alignment of the sun's magnetic and rotational axis (Pastor Yabar et al. 2015) using line-of-sight HMI magnetograms for a 5 year period starting from April 2010. Apart from finding a monthly oscillation at all solar latitudes which they attributed to a non-alignment in the solar magnetic and rotational axis, their data also indicated the time of occurrence of the solar polar field reversals to be in $\sim$ Feb. 2013 for the northern hemisphere and $\sim$ April 2014 for the southern Hemisphere (see Fig. 2 of Pastor Yabar et al. 2015).

The heliospheric signatures of this unusual polar reversal pattern, studied using synoptic maps of solar wind velocities, has shown the development of polar coronal holes in the northern hemisphere as late as in October 2016. Since the heliospheric measurements of solar wind velocities are made in the corona at a height higher than that of the $17 \mathrm{GHz}$ microwave emission, it is, thus, expected that the time of the polar reversal pattern could be later than the time of polar reversal from the $17 \mathrm{GHz}$ microwave brightness enhancement.

The polar reversal pattern, in cycle 24 , in the solar northern hemisphere was very unusual and probably unprecedented. A field reversal or zero crossings of the magnetic field first occurred in June 2012. However, this was followed by a long period when the strength of the field did not increase and remained nearly zero until November 2014, after which it showed a clear and unambiguous increase. The southern hemisphere, on the other hand, showed only a single unambiguous zero crossing or field reversal in November 2013, as can be seen in Fig. 1. The hemispheric asymmetry of polar field reversal is well known and well discussed by Svalgaard \& Kamide (2013) wherein, the authors attributed such hemispheric asymmetry to the asymmetry in solar activity in both the hemispheres. Our study of the variation of toroidal magnetic fields showing a hemispheric asymmetry in solar activity in the sunspot belt regions lends support to the report by Svalgaard \& Kamide (2013). The unusual pattern of field reversals in the northern hemisphere in cycle 24 can be attributed to multiple surges of solar activity after 2012 that carried the wrong magnetic flux to the solar north pole as seen by the latitudinal field profiles in the lower panel of Fig. 3 in this study. It is important to note here that a clean or unambiguous field reversal can occour when the magnetic flux transported to the poles has a polarity that is the opposite to that of the incumbent polarity. It is to be noted that the present study very clearly shows the occurrence of multiple such surge activity in the northern hemisphere, having the same polarity as the incumbent polarity in the north during cycle 24 .

As opposed to an early study, carried out using $17 \mathrm{GHz}$ microwave imaging observations (Gopalswamy et al. 2016), that suggested that the zero field condition in the northern hemisphere lasted until late 2015, our study of the latitudinal field profiles from the magnetic butterfly diagram as well as the unsigned polar field strength poleward of $78^{\circ}$ show that the zero field condition in the northern hemisphere existed for nearly 2.5 years from $\sim$ June 2012 until $\sim$ Nov. 2014 .

Our study shows that the polar field strength during cycle 24 was comparatively weaker than in the previous cycle 23 . Also, the polar field strength in cycle 23 had been weaker than the earlier cycles 21-22 (Wang et al. 2009; Janardhan et al. 2010). Thus, it is clear that the polar field strength has been steady declining from cycle 21 to 24 . Janardhan et al. (2010) showed a very good correlation of polar field strength in the latitude range $78^{\circ}-90^{\circ}$ with the meridional flow speed, reported by Hathaway \& Rightmire (2010) in cycle 23. In the present study, we also found a similar temporal behaviour of the polar field strength in the latitude range $78^{\circ}-90^{\circ}$ during cycle 24 implying that the polar field strength and the meridional flow speed are correlated. Since the polar field strength during cycle 24 is seen to be weaker in comparison to the earlier cycle 23 , we, thus, believe that the meridional flow speed could have been faster in cycle 24 than cycle 23 leading to a weakening of the polar field strength in cycle 24 . Further, the considerably weaker polar field strength seen in the northern hemisphere as compared to the southern hemisphere implies that the meridional flow speed in cycle 24 is hemispherically asymmetric with a faster meridional flow in the north than the south. As a result of the faster meridional flow in the north, surge activitie of alternate polarities are noticed in the northern hemisphere, which has, in turn, caused the prolonged zero-field conditions in the north by reducing the field strength due to multiple flux cancellations.

It is known that the strength of the polar field can be used as precursor for predicting the strength of the upcoming cycle (Schatten \& Pesnell 1993; Schatten 2005). The weaker polar field strength of cycle 24 implies a weaker cycle 25 in keeping with the flux transport dynamo model prediction of Choudhuri et al. (2007) wherein, the authors used the axial dipole moments of the previous cycle to successfully predict the amplitude of the next cycle. Using the long term variations in unsigned high-latitude $\left(45^{\circ}-78^{\circ}\right)$ solar magnetic fields (Janardhan et al. 2015a), a prediction of $\sim 62 \pm 12$ has been made for the maximum sunspot number for solar cycle 25 in the old unmodified sunspot number scale indicating a weaker cycle 25 than cycle 24 . This study lends support to the prediction that the amplitude of the next cycle 25 will be weaker than the current cycle 24 . A similar interpretation, that the cycle 25 will be comparatively weaker than the cycle 24 , has also been obtained by Upton \& Hathaway (2014).

The upper panel of Fig. 7 shows by the filled dots and open circles, the unsigned values of solar polar fields for the northern and southern hemispheres, obtained from line-of-sight $\mathrm{NSO} / \mathrm{KP}$ magnetograms, in the latitude ranges $45^{\circ}-78^{\circ}$. Similarly, the lower panel shows the data for the latitude range $0^{\circ}-45^{\circ}$. The data points shown start from 1985 (cycle 22) and cover the period until the end of 2017 in cycle 24 . Overplotted on the data points are solid curves in red (northern) and blue (southern) showing the smoothed variations of the data in the two hemispheres. Earlier studies (Bisoi et al. 2014a; Janardhan et al. 


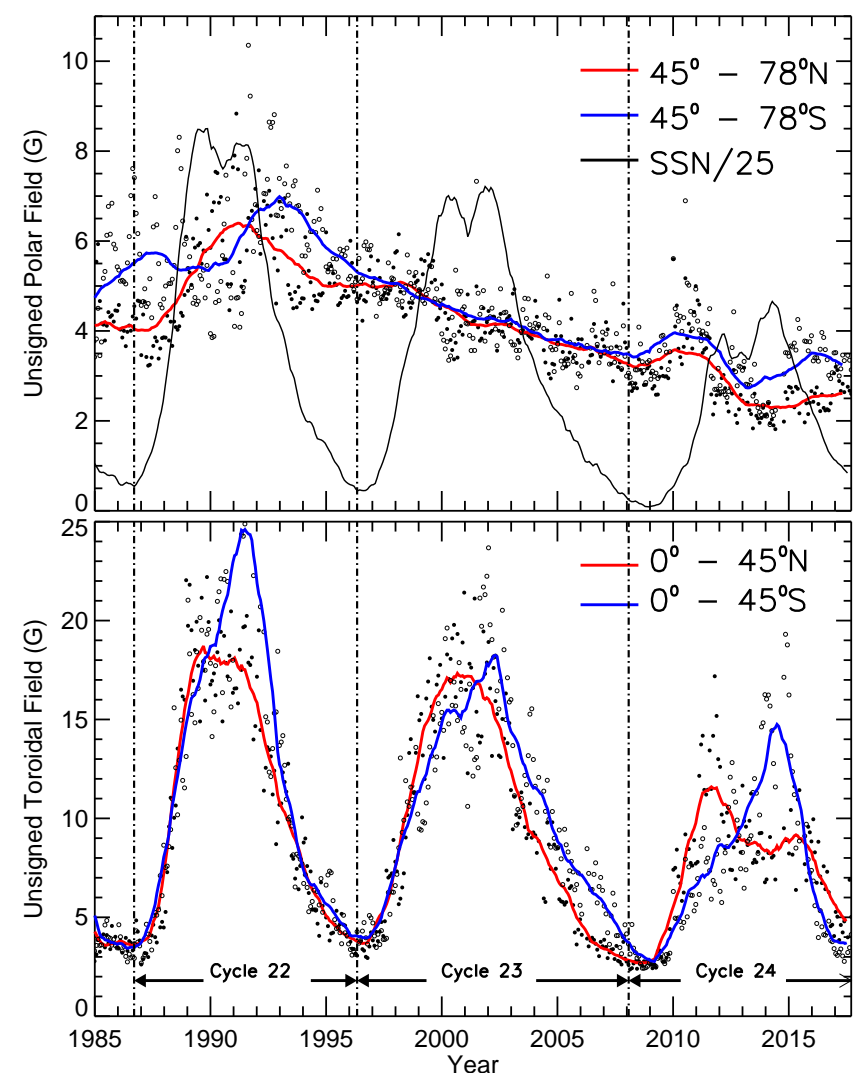

Fig. 7. Upper panel shows, respectively, by the filled dots and open circles, the unsigned values of solar polar fields for the northern and southern hemispheres, obtained from NSO/KP magnetograms, in the latitude ranges $45^{\circ}-78^{\circ}$, for solar cycles $22-24$. Also, overplotted are the solid curves in red and blue showing the smoothed variations of data points for the northern and southern hemispheres, respectively. Lower panel: same for the unsigned values of toroidal fields in the latitude ranges $0^{\circ}-45^{\circ}$. The smoothed total sunspot number, scaled down by a factor of 25 , is shown in solid black curve in the upper panel. The vertical dotted lines demarcate the solar cycle periods.

2015a) have reported a steady 20 year decline, starting from the mid-1990's to the end of 2014 of solar photospheric fields in the latitude ranges $45^{\circ}-78^{\circ}$. It is evident from a careful examination of Fig. 7 (upper panel) that the photospheric fields continuing to decline in the northern hemisphere while the field strength in the southern hemisphere has at least partially recovered and shown an increase since June 2014. It can be seen from the lower panel of Fig. 7 that the photospheric fields, at the end of 2017, have already approached the minimum in the southern hemisphere while in the northern hemisphere it is still declining and has yet to approach the minimum. This asymmetry in the decline in both hemispheres and the continuation of photospheric field decline in the north explain the prolonged zero-field condition and delayed polar reversal of the northern hemisphere. Due to this the overall trend is still that of a decline and one would expect that this would continue at least until 2020, the expected minimum of the current cycle 24 .

The study of solar photospheric fields and a continuation of the study of solar polar field reversal process is, therefore, of utmost importance in understanding the sun, the solar dynamo process, the solar wind and space weather, more so because of the fact that such a situation on the sun, when solar photospheric fields have been steadily declining for nearly 25 years now and there is speculation that we are probably on the verge of a grand minimum akin to the Maunder minimum (Janardhan et al. 2015a,b;
Sánchez-Sesma 2016), is unique and probably unprecedented since systematic solar observations began four centuries ago.

Acknowledgements. We acknowledge the free data use policy of NSO/KP, NSO/SOLIS, SoHO/MDI, SDO/HMI and WDC-SILSO and of the Royal Observatory, Belgium facilities. JP, DR and KF acknowledge the ISEE International Collaborative Research Program for support in executing this work. SKB is supported by PIFI program of the Chinese Academy of Sciences (Project Number: 2015PM066) and NSFC (Grant no. 117550110422).

\section{References}

Altrock, R. C. 2011, Sol. Phys., 274, 251

Ananthakrishnan, S., Balasubramanian, V., \& Janardhan, P. 1995, Space Sci. Rev., 72, 229

Babcock, H. D. 1959, ApJ, 130, 364

Babcock, H. W. 1961, ApJ, 133, 572

Balasubramanian, V., Janardhan, P., Srinivasan, S., \& Ananthakrishnan, S. 2003, J. Geophys. Res., 108, 1121

Benevolenskaya, E. E. 2004, A\&A, 428, L5

Bisoi, S. K., Janardhan, P., Chakrabarty, D., Ananthakrishnan, S., \& Divekar, A. 2014a, Sol. Phys., 289, 41

Bisoi, S. K., Janardhan, P., Ingale, M., et al. 2014b, ApJ, 795, 69

Choudhuri, A. R., Chatterjee, P., \& Jiang, J. 2007, Phys. Rev. Lett., 98, 131103 de Toma, G. 2011, Sol. Phys., 274, 195

Dikpati, M., de Toma, G., Gilman, P. A., Arge, C. N., \& White, O. R. 2004, ApJ, 601, 1136

Dikpati, M., Gilman, P. A., de Toma, G., \& Ulrich, R. K. 2010, Geophys. Res. Lett., 37, 14107

Domingo, V., Fleck, B., \& Poland, A. I. 1995, Sol. Phys., 162, 1

Durrant, C. J., \& Wilson, P. R. 2003, Sol. Phys., 214, 23

Fox, P., McIntosh, P., \& Wilson, P. R. 1998, Sol. Phys., 177, 375

Fujiki, K., Tokumaru, M., Hayashi, K., Satonaka, D., \& Hakamada, K. 2016, ApJ, 827, L41

Gopalswamy, N., Yashiro, S., Mäkelä, P., et al. 2012, ApJ, 750, L42

Gopalswamy, N., Yashiro, S., \& Akiyama, S. 2016, ApJ, 823, L15

Hathaway, D. H., \& Rightmire, L. 2010, Science, 327, 1350

Hewish, A., Scott, P. F., \& Wills, D. 1964, Nature, 203, 1214

Howard, R. 1972, Sol. Phys., 25, 5

Janardhan, P., \& Alurkar, S. K. 1993, A\&A, 269, 119

Janardhan, P., Balasubramanian, V., Ananthakrishnan, S., et al. 1996, Sol. Phys., 166,379

Janardhan, P., Bisoi, S. K., \& Gosain, S. 2010, Sol. Phys., 267, 267

Janardhan, P., Bisoi, S. K., Ananthakrishnan, S., Tokumaru, M., \& Fujiki, K. 2011, Geophys. Res. Lett., 38, L20108

Janardhan, P., Bisoi, S. K., Ananthakrishnan, S., Sridharan, R., \& Jose, L. 2015a, Sun and Geosphere, 10, 147

Janardhan, P., Bisoi, S. K., Ananthakrishnan, S., et al. 2015b, J. Geophys. Res., 120,5306

Jian, L. K., Russell, C. T., \& Luhmann, J. G. 2011, Sol. Phys., 274, 321

Karna, N., Hess Webber, S. A., \& Pesnell, W. D. 2014, Sol. Phys., 289, 3381

Kirk, M. S., Pesnell, W. D., Young, C. A., \& Hess Webber, S. A. 2009, Sol. Phys., 257, 99

Kojima, M., Tokumaru, M., Watanabe, H., et al. 1998, J. Geophys. Res., 103, 1981

Krieger, A. S., Timothy, A. F., \& Roelof, E. C. 1973, Sol. Phys., 29, 505

Leighton, R. B. 1969, ApJ, 156, 1

Lemen, J. R., Title, A. M., Akin, D. J., et al. 2012, Sol. Phys., 275, 17

Makarov, V. I., Fatianov, M. P., \& Sivaraman, K. R. 1983, Sol. Phys., 85, 215

Moran, P. J., Ananthakrishnan, S., Balasubramanian, V., et al. 2000, Ann. Geophys., 18, 1003

Muñoz-Jaramillo, A., Sheeley, N. R., Zhang, J., \& DeLuca, E. E. 2012, ApJ, 753, 146

Nolte, J. T., Krieger, A. S., Timothy, A. F., et al. 1976, Sol. Phys., 46, 303

Pastor Yabar, A., Martínez González, M. J. \& Collados, M. 2015. MNRAS, 453, L69

Petrie, G. J. D. 2012, Sol. Phys., 281, 577

Sánchez-Sesma, J. 2016, Earth Syst. Dyn., 7, 583

Schatten, K. 2005, Geophys. Res. Lett., 32, 21106

Schatten, K. H., \& Pesnell, W. D. 1993, Geophys. Res. Lett., 20, 2275

Sun, X., Hoeksema, J. T., Liu, Y., \& Zhao, J. 2015, ApJ, 798, 114

Svalgaard, L., \& Kamide, Y. 2013, ApJ, 763, 23

Upton, L., \& Hathaway, D. H. 2014, ApJ, 780, 5

Wang, Y.-M., Nash, A. G., \& Sheeley, N. R., Jr. 1989, ApJ, 347, 529

Wang, Y.-M., Robbrecht, E., \& Sheeley, N. R., Jr. 2009, ApJ, 707, 1372

Webb, D. F., Davis, J. M., \& McIntosh, P. S. 1984, Sol. Phys., 92, 109

Zirker, J. B. 1977, Rev. Geophys. Space Phys., 15, 257 